\title{
An Experimental Examination of Mobile Cloud Computing and IOT: Application, Techniques and Issues
}

\author{
Taranpreet Kaur
}

\begin{abstract}
A technology in which voice, video and data can transfer through a computer or any wireless device is known as mobile computing. There is no need to connect to a fix physical link in mobile computing. By using the cloud computing mobile computing the powerful mobile applications can build.

The approach of mobile cloud computing allows the developers to design the applications especially for mobile users. And the applications are not bounded with the memory capacity, computing process and operating system of Smartphone. From a remote web server through mobile browser the mobile cloud computing centered simply accesses. On the recipient Smartphone it is not necessary to install the client application.
\end{abstract}

Index Terms: mobile computing; mobile cloud computing; mobile operating system; Smartphone.

\section{INTRODUCTION}

To interact with the world in recent days it have to develop a technique for shifting the technologies which can change the way of program. In these techniques mobile computing and cloud computing are two prominent research files that can produce the effective impact. To promote smart phone it is necessary to develop a network for Internet-enabled devices, Internet of Things (IoT) is also a solution for getting the better result [8]. Detailed description about mobile cloud computing and IOT are described in next sections.
Revised Manuscript Received on July 22, 2019.

Taranpreet Kaur, Asst. Prof., Dept. of Computer Science, Mata Gujri College, Fategarh Sahib,Panjab,India

\section{MOBILE COMPUTING}

The set of products, operational strategies, procedures, IT technologies and services through which user can access the information, related resources, capabilities and computation on mobile is known as mobile computing. Mobile technologies are not restricted to any geographic location because it can refer as access in motion.

Many mobile devices can connect with wi-fi or wireless technology or with local area networks. They can also connect through wide area networks.

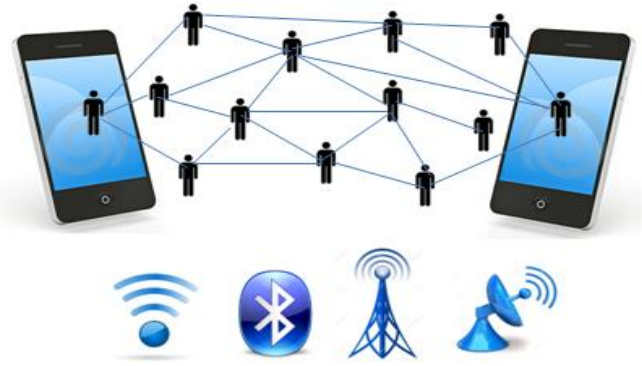

Figure 1: Mobile Computing

a. Mobile Computing advantages

Some advantages of mobile computing are:

1. Personalization: it possible to create the mobile application for personal use.

2. Social connection: by using several mobile applications it is possible to interact with many users by using internet

3. Connectivity: by using mobile applications it is possible to connected to many resources at any time.

\section{b. Mobile Computers Types}

Since 1990's there have been developed so many mobile computers. Some of them are:

1. Smartphone

2. Laptop

3. Mobile data terminal (MDT)

4. Tablet personal computer

5. Personal digital assistant (PDA)

\section{Mobile Cloud Computing}

To develop a strong computational research for cloud computing users, network operators and mobile users the mobile cloud computing is used as a combination of mobile computing and cloud computing [1][2][3]. To give the user a strong experience the mobile cloud computing goal is to develop 
strong mobile application on a plethora of mobile devices [4]. The mobile cloud computing provide business opportunities for both mobile networks and cloud service providers [5] [6]. The mobile cloud computing can defined as a technology that enables the various resources of mobile and cloud computing towards unrestricted storage, mobility and functionality of mobile devices at anytime, anywhere with the help of internet or Ethernet [7].

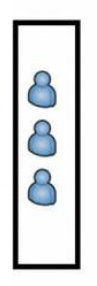

Users

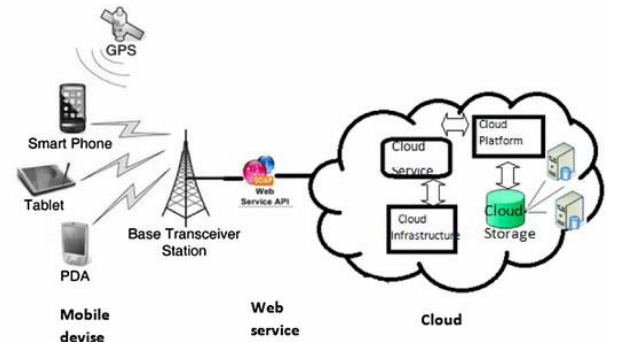

Figure 2: Mobile Cloud Computing

\section{a. Mobile Cloud Computing Applications}

Mobile cloud computing applications are refers to as a software program that can use by several portable computing apparatus over the internet. Mobile cloud app and mobile web apps are two types of applications that are almost same. In both applications data stored externally and can access through a browser using internet. Both applications run on external server of mobile devices [8].

\section{b. Smart phone with cloud computing}

For defining the smart phone these are several ways. Characteristics of Smart phone are given below:

1. It is necessary for the smart phone to run the applications that are stored.

2. Smart phone have option to send and receive messages

3 . There should be a recognizable operating system of smart phone

4. Smart phone should have a recognizable internet connection

5. Smart phone should provide an advance feature of calling

In the recent scenario of cloud, it is based on the strategy that large size information devices can provide information and updates to both old and new customers. Now it has seen that cloud has the clients like all hand held devices, mobile phone and tablets. If a phone is developed in a way that for large distributed network every phone behaves likes a node then the overall computer will have much more power and capability [9].

\section{c. Benefits and services of Mobile Cloud computing}

Some benefits and services of mobile cloud computing is given below:

1. The business that are using mobile cloud computing can reduce the overall cost

2. In this filed more network provider can join with their set up

3. Because of portability the owner of enterprises can get mobile cloud computing

4. By using mobile computing developer can reach to large market
5. By using mobile cloud computing the mobile user can access more features on their mobile phones.

\section{Role of Cloud Computing IN IOT}

The way in which daily routine work are completed are generally transformed by using Internet of Things (IoT). It is possible in recent days that a people can control their cooling devices with the help of mobile. Earlier this can do by SMS but by use of internet it can be easier now.

The cloud computing provide database storage, IT resources, applications, computing power so it is require to in many areas to combine with IoT. The cloud computing and IoT features are shown in table given below:

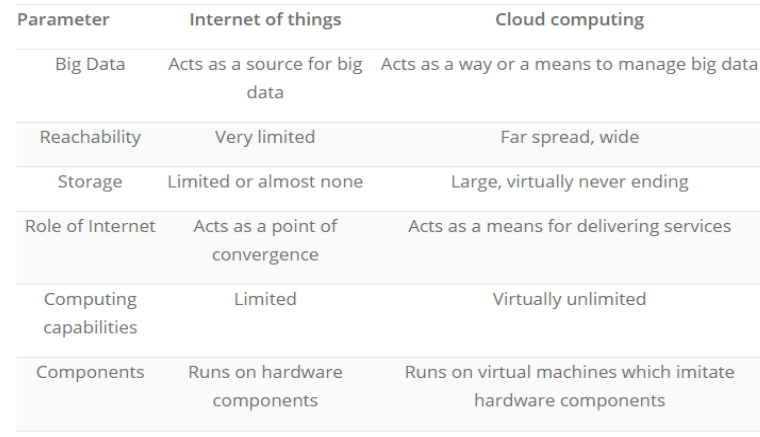

Table 1: internet of things and cloud computig parameters

Internet of things and cloud computing both work for enhancing the efficiency in daily routine work and both have a connection that complement to each other. The IoT provide lots of data and cloud computing provide a way to pass that data. To provide pay-as-you-use model many service providers off cloud take benefits of this [11].

\section{a. Cloud Computing Categories}

The cloud computing generally use for photos, applications, videos, delivering data. It has the cloud that act as center of data on internet. The IBM divided cloud computing in six categories as follows:

1. Public Cloud: this is basically develop for using data publically. Any company can own and operate this space and it give quick access to user on public network.

2. Private Cloud: it is similar as public cloud but only one entity can access the data at one time. it can be specifically design for particular organization or system.

3. Hybrid Cloud: in this type of cloud it found like private cloud but access the public cloud.

4. Software as a service (SaaS): in this type of cloud the applications that based on cloud run on off site of computer. Other companies and people who connect to computer of user can own these devices through web server.

5. Platform as a service (PaaS): to create and deliver the applications which based on cloud all the necessary services provided by this type of cloud. So there is no need to purchase hosting, software, hardware etc.

6. Infrastructure as a service (IaaS): on the basis of per user IaaS provides companies with networking, data centers, storage, and servers.

\section{b. IOT Cloud Applications}

The real action takes place on cloud. To and from the sensor or gateway APIs and other interfaces handle the commands 
and data along with IoT cloud application [12]. To stored and read data accurately different APIs need to be integrated. For development of cloud application best practices are:

1. Design of database

In any IoT creation data storage is a major task because IoT stored lots of data. Analysis of sensor node and according to design a data base include by best practices. It is also optimize the data management.

2. Security of application

Security is major concern in IoT cloud computing because there is always a possibility of hack the data. The network across which data packet sent should encrypted first and it enabled TLS/SSL certificate so the devices and sensors which access the remotely can averted.

\section{Cloning of application}

In case of heavy traffic the cloning application help to avoid overloading of data. This application is must enabled on the IoT cloud application because this the best way to handle increased traffic.

\section{Server Scalability}

The server has an option of auto scaling depend on the projected and current number of end user. For this feature service like AWS-EC2 can enabled.

\section{c. Types of Cloud Computing Models for IoT Solutions

\begin{tabular}{|c|c|}
\hline \multicolumn{2}{|c|}{ Cloud Computing Models } \\
\hline Infrastructure as a Service & Platform as a Service \\
\hline $\begin{array}{l}\text { - It offers virtual servers and } \\
\text { storage to the enterprises. } \\
\text { Basically, it enables the access to } \\
\text { the networking components like } \\
\text { computers, data storage, network } \\
\text { connections, load balancers, and } \\
\text { bandwidth. } \\
\text { - Increasing critical data within the } \\
\text { organization lead to the security } \\
\text { vulnerabilities and laaS can help in } \\
\text { distributing the critical data at } \\
\text { different locations virtually (or } \\
\text { can be physical) for improving the } \\
\text { security. }\end{array}$ & $\begin{array}{l}\text { - It allows companies to create } \\
\text { software and applications from } \\
\text { the tools and libraries provided by } \\
\text { the cloud service providers. } \\
\text { - It removes the basic needs of } \\
\text { managing hardware and operating } \\
\text { systems and allows enterprises to } \\
\text { focus more on the deployment and } \\
\text { management of the software or } \\
\text { applications. } \\
\text { - It reduces the worry of } \\
\text { maintaining the operating system, } \\
\text { capacity planning, and any other } \\
\text { heavy loads required for running } \\
\text { an application. }\end{array}$ \\
\hline
\end{tabular}

Table 2: cloud computing model for IOT

\section{d. Benefits of Cloud in an IoT System}

There are a several benefits of using a cloud in Iot system.

Some of them are as follows:

1. Advance monitoring and analytics

In order to feel the seamless experience for user constant monitoring and analysis are very necessary. In advance development of cloud applications this type of services enabled with cloud.

\section{2. inter-device connectivity is smoother}

the sensor is communicative with user as well as with others in an IoT. Along with IoT gateway the cloud application check that different actuators and sensor without any disturbance should able to communicate to each other.

\section{e. Challenges in IOT cloud computing}

There are many challenges arise due to rapid growth in IoT cloud applications. Researches do many works on this topic but there is still need to develop research to face the many challenges. In IoT management there are several challenges arise. They are:

1. Adversaries of internet

2. Heterogeneity and device mobility

3. Data management

4. Data storage

5. Integrity

6. Privacy

7. Confidentiality

\section{DEVELOPING THE ENTERPRISE ARCHITECTURE OF Mobile ClOUd COMPUTING AND IOT}

To develop a Enterprise Mobile Cloud Application MobileStack is used. For last 20 years it provides a strong background in wireless and mobile applications. For fulfill the requirement of an enterprise it has to develop an an enterprise mobile cloud application.

By integrating various components of mobile cloud services MobileStack has developed an Enterprise-MobileStack services platform.

\section{Conclusion}

A technology in which voice, video and data can transfer through a computer or any wireless device is known as mobile computing. There is no need to connect to a fix physical link in mobile computing. By using the cloud computing mobile computing the powerful mobile applications can build.

Mobile cloud computing focused are for the most part gotten to by means of a versatile program from a remote web server, ordinarily without the requirement for introducing a customer application on the beneficiary phone. To build up a solid computational research for distributed computing clients, organize administrators and versatile clients the portable distributed computing is utilized as a blend of portable figuring and cloud computing. The manner by which day by day schedule work are finished are commonly changed by utilizing Internet of Things (IoT). It is conceivable as of late that a people can control their cooling gadgets with the assistance of versatile.

\section{REFERENCES}

1. Khan, A. u R.; Othman, M.; Madani, S. A.; Khan, S. U. (2014-01-01). "A Survey of Mobile Cloud Computing Application Models". IEEE Communications Surveys and Tutorials. 16 (1): 393-413. CiteSeerX 10.1.1.402.1725.

2. Abolfazli, Saeid; Sanaei, Zohreh; Ahmed, Ejaz; Gani, Abdullah; Buyya, Rajkumar (1 July 2013). "Cloud-Based Augmentation for Mobile Devices: Motivation, Taxonomies, and Open Challenges". IEEE Communications Surveys \& Tutorials. 99 (pp): 337-368.

3. Fangming Liu, Peng Shu, Hai Jin, Linjie Ding, Jie Yu, Di Niu, Bo Li, "Gearing Resource-Poor Mobile Devices with Powerful Clouds: Architecture, Challenges and Applications", IEEE Wireless Communications Magazine, Special Issue on Mobile Cloud Computing, vol. 20, no. 3, pp.14-22, June, 2013.

4. Abolfazli, Saeid; Sanaei, Zohreh; Gani, Abdullah; Xia, Feng; Yang, Laurence T. (1 September 2013). "Rich Mobile Applications: Genesis, taxonomy, and open issues". Journal of Network and Computer Applications. 40: 345-362.

5. Khan, A. u R.; Othman, M.; Xia, F.; Khan, A. N. (2015-05-01). "Context-Aware Mobile Cloud Computing and Its Challenges". IEEE Cloud Computing. 2 (3): 42-49. doi:10.1109/MCC.2015.62. ISSN 2325-6095.

6. Dinh, Hoang T. (2013). "A survey of mobile cloud computing: architecture, applications, and approaches". $\quad$ Wireless 
Communications and Mobile Computing. 13 (18): 1587-1611.

7. Sanaei, Zohreh; Abolfazli, Saeid; Gani, Abdullah; Buyya, Rajkumar (1 January 2013). "Heterogeneity in Mobile Cloud Computing: Taxonomy and Open Challenges" (PDF). IEEE Communications Surveys \& Tutorials (99): 1-24.

8. Siegel, J. E. (2016). Data proxies, the cognitive layer, and application locality: enablers of cloud-connected vehicles and next-generation internet of things (Doctoral dissertation, Massachusetts Institute of Technology).

\section{AUTHORS PROFILE}

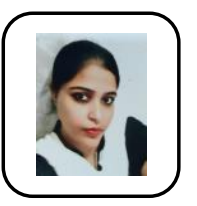

Taranpreet Kaur's educational qualification is MCA, M Phil and having 14 years of teaching experience as Assistant Professor, Dept. of Computer Science. She has attended three refresher \& orientation program at Punjabi University, Patiala, six workshops, and published eighteen research papers (Two Scopus Indexed) in national and international conferences. She has written two book chapters and two books and two are in progress. 